\title{
Native-State Structural Analysis of Respiratory Syncytial Virus.
}

Zunlong Ke ${ }^{1}$, Rebecca S. Dillard ${ }^{2}$, Cheri M. Hampton ${ }^{2}$, Rachel E. Storms ${ }^{2}$, Joshua D. Strauss ${ }^{2}$, and E. R. Wright $^{2,3}$

${ }^{1 .}$ School of Biology, Georgia Institute of Technology, Atlanta, Georgia, USA.

2. Emory University School of Medicine, Division of Pediatric Infectious Disease, and Children's Healthcare of Atlanta, Atlanta, Georgia, USA

${ }^{3 .}$ Robert P. Apkarian Integrated Electron Microscopy Core, Emory University, Atlanta, Georgia, USA

Human respiratory syncytial virus (hRSV) is an enveloped RNA virus that belongs to Paramyxoviridae family. HRSV is the most common cause of bronchiolitis and pneumonia in young children in the United States, and severe infections of infants and immuno-compromised adults can lead to death. In order to characterize the molecular mechanisms underlying hRSV assembly and viral and host cellular protein dynamics at high spatiotemporal resolution, we use cryo-electron tomography (cryo-ET) and cryo-correlative light and electron microscopy (cryo-CLEM) to examine hRSV-infected cells.

Our cryo-ET studies have demonstrated that the native-state morphology of hRSV is filamentous from hRSV-infected HeLa cells, as well as in four other lung-derived cell lines (Figure 1), when compard to the pleomorphic structure of hRSV present in purified vius particle preparations [1]. The viral filament length varies from $\sim 1$ to $8 \mu \mathrm{m}$. We are able to resolve the overall three-dimensional (3D) structure and relative locations of the viral structural proteins in assembling and released viral filaments among the five cell lines tested, including the surface glycoproteins, the matrix protein $(\mathrm{M})$, the ribonucleoprotein complex (RNP), and the linker protein M2-1 between M and RNP [1]. We have observed two arrangements of the surface glycoproteins: ordered rows and a hexameric lattice. Subtomogram averaging of the glycoprotein hexamers revealed center to center spacing between neighboring hexamer units is $\sim 12 \mathrm{~nm}$. We also investigated the structure of several recombinant hRSV (rRSV) strains. Analysis of the glycoproteins from one rRSV strain using subtomogram averaging indicated that the $F$ glycoprotein retained a prefusion state. The matrix protein appears to form oligomers at sites of assembly and filament elongation. We have captured stages of hRSV assembly, including assembly initiation at the plasma membrane, viral filament elongation, and scission. In addition, we have resolved early assembly events using cryo-immunogold labeling [2].

Cryo-ET combined with native immunolabeling of surface glycoproteins will aid in distinguishing prefusion and postfusion glycoprotein conformations, and determining the order of viral protein recruitment to sites of assembly. We frequently resolve cellular factors, such as cytoskeletal networks and ribosomes, near and within the assembling viral filaments (Figure 1). Future studies will determine the biological significance of ordered glycoprotein arranegements and the role of matrix protein oligomers in the assembly process. We propose that the fusion glycoprotein initiates hRSV assembly and the matrix protein oligomers facilitate elongation by recruiting the RNP complex and driving subsequent filament elongation. We anticipate that our proposed model for hRSV will contribute to structure-directed anti-viral drug design and vaccine development, and potentially other paramyxoviruses. 
References:

[1] G. Kiss, et al. Journal of Virology 88 (2014), p. 7602.

[2] H. Yi, et al. Journal of Histochemistry \& Cytochemistry 63 (2015), p. 780.

[3] This research was supported by funds from Emory University, Children's Healthcare of Atlanta, the Emory Center for AIDS Research, the Georgia Research Alliance, Human Frontiers Science Program, National Institutes of Health (5R21AI101775, 1R01GM114561, 3R01GM104540-03S1), and the National Science Foundation (0923395) to E.R.W, and S10 RR025679 to P.W.S. All EM data was collected at the Emory University Robert P. Apkarian Integrated Electron Microscopy Core.

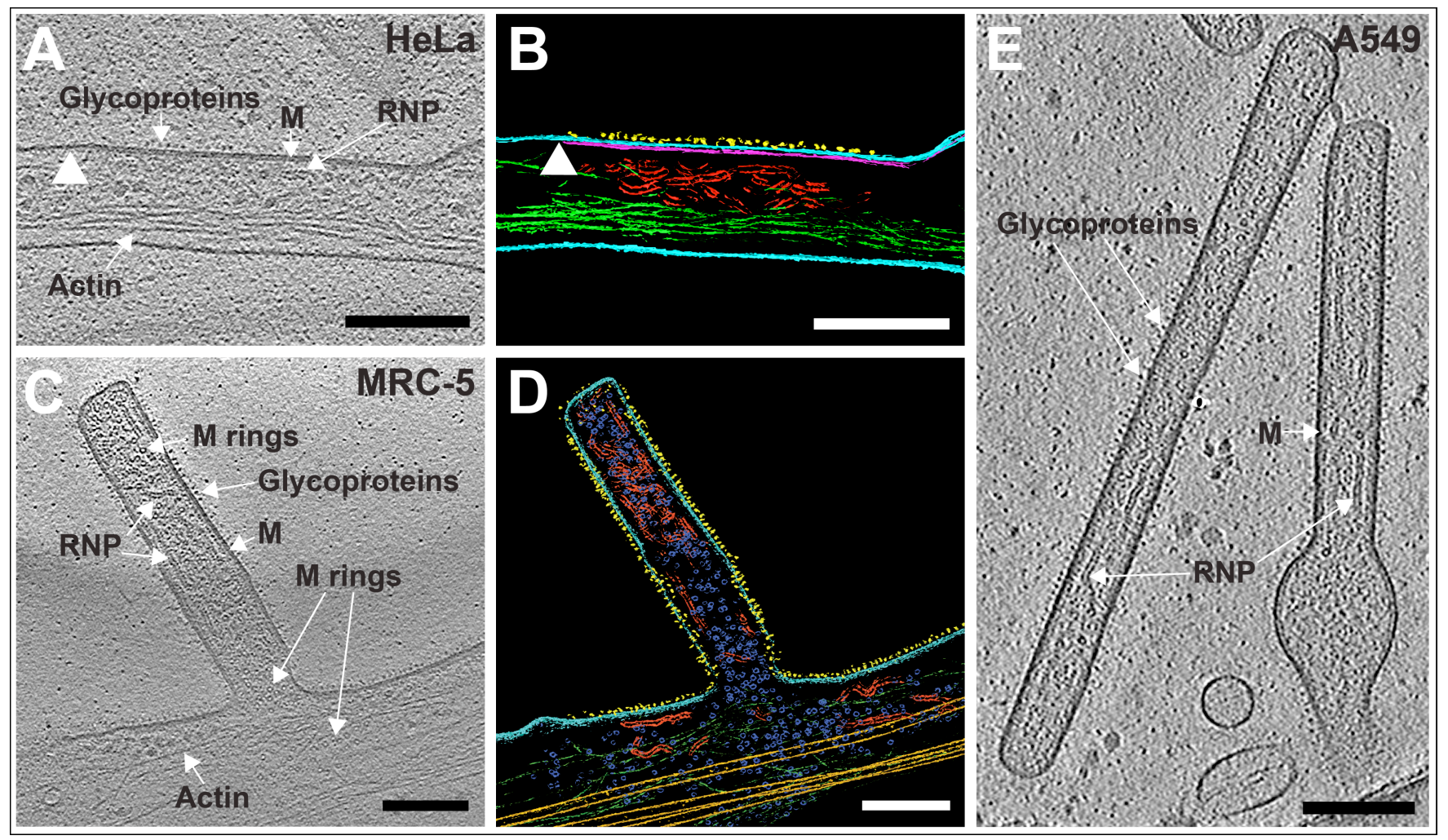

Figure 1. Tomographic slices (A, C, E) and segmentation views (B, D) from hRSV infected cells. (A-B) Initial site of virus assembly from hRSV-infected HeLa cell. Tomographic slice (A) and corresponding segmentation view (B). (C-D) Matrix protein rings in assembling viral filaments from hRSV-infected MRC-5 cell. Tomographic slice (C) and corresponding segmentation view (D). (E) Tomographic slice of hRSV filaments that budded from an infected A549 cell. For segmentation view (B, D): membrane (cyan); glycoproteins (yellow); RNP (red); matrix protein rings (blue); actin (green), microtubules (gold). Scale bars $200 \mathrm{~nm}$. 